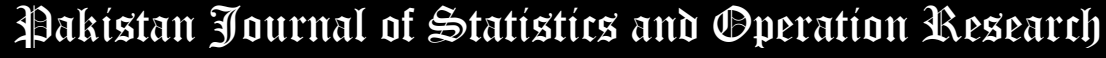

\section{Statistical Inference for Inverted Kumaraswamy Distribution Based on Dual Generalized Order Statistics}

\author{
G. R. AL-Dayian ${ }^{1}$, A. A. EL-Helbawy ${ }^{2 *}$ and A. M. Abd AL-Fattah ${ }^{3}$ \\ * Corresponding Author aah_elhelbawy@ hotmail.com
}

1. Statistics Department, Faculty of Commerce, AL-Azhar University (Girls' Branch), Cairo, Egypt, aldayian@yahoo.com

2. Statistics Department, Faculty of Commerce, AL-Azhar University (Girls' Branch), Cairo, Egypt, aah_elhelbawy@hotmail.com

3. Statistics Department, Faculty of Commerce, AL-Azhar University (Girls' Branch), Cairo, Egypt, asmaa.stat@gmail.com

\begin{abstract}
In this paper, the shape parameters, reliability and hazard rate functions of the inverted Kumaraswamy distribution are estimated using maximum likelihood and Bayesian methods based on dual generalized order statistics. The Bayes estimators are derived under the squared error loss function as a symmetric loss function and the linear-exponential loss function as an asymmetric loss function based on dual generalized order statistics. Confidence and credible intervals for the parameters, reliability and hazard rate functions are obtained. All results are specialized to lower record values, also a numerical study is presented to illustrate the theoretical procedures.
\end{abstract}

Key Words: Inverted Kumaraswamy distribution; Maximum likelihood estimation; Bayesian estimation; Lower records; Monte Carlo simulation.

\section{Mathematical Subject Classification: 62F10, 62F15}

\section{Introduction}

The concept of generalized order statistics (gos) was introduced by Kamps (1995) as a unified models for ordered random variables which produce several models as a special case. These models play an important role in statistics in general and in reliability theory and life testing in particular. Since its inception gos has attracted number of statisticians as distribution specific results obtained for gos can be used to obtain the results for other models of ordered random variables as special case. The random variables that are decreasingly ordered cannot be integrated into this framework. Burkschat et al. (2003) studied the dual generalized order statistics (dgos) that enables a common approach to descending ordered random variables as reversed ordered order statistics, lower record models and lower Pfeifer records. Some applications in reliability theory, such as, times of failure of technical components or systems, the failure of some components of the system can be more or less strongly influence life-length distribution of the remaining components in the system. Also as a model for successively largest insurance claims, highest water-levels or highest temperatures. For more details, see Burkschat et al. (2003), Khaledi and Kochar (2005), Jaheen and Al Harbi (2011), Khan and Khan (2012), MirMostafaee et al. (2016) and Mahdizadeh (2016).

Let $X(1, n, m, k), X(2, n, m, k), \ldots, X(n, n, m, k)$ are $n$ dgos from an absolutely cumulative distribution function (cdf) with corresponding probability density function (pdf). Then, the joint pdf has the form

$f^{X(1, n, m, k), \ldots, X(n, n, m, k)}\left(x_{(1)}, \ldots, x_{(n)}\right)=k\left(\prod_{j=1}^{n-1} \gamma_{j}\right)\left[\prod_{i=1}^{n-1}\left(F\left(x_{(i)}\right)\right)^{m} f\left(x_{(i)}\right)\right]\left(F\left(x_{(n)}\right)\right)^{k-1} f\left(x_{(n)}\right)$, 
where $F^{-1}(1) \geq x_{(1)} \ldots \geq x_{(n)} \geq F^{-1}(0), n \in N, k \geq 1, m_{1}, \ldots, m_{n-1}=m$,

$m \in \mathbb{R}$ and $\gamma_{r}=k+(n-r)(m+1) \geq 1, \quad$ for all $1 \leq r \leq n$.

For more details of dgos, see Ahsanullah (2004), Khan and Kumar (2010), Athar and Faizan (2011), Tavangar (2011), Mahmoud et al. (2014), Kumari and Pathak (2014) and Kim et al. (2016). Statistical modeling using new distributions has been largely studied in recent years, for an example see MirMostafaee et al. (2015) and MirMostafaee et al. (2017).

Kumaraswamy (1980) constructed Kumaraswamy (Kum) distribution which is applicable to many natural phenomena whose outcomes have lower and upper bounds, such as heights of individuals, scores obtained in a test, atmospheric temperatures and hydrological data. Some papers deal with different methods of generalization for the Kum distribution, see Cordeiro et al. (2010) and Barreto-Souza and Lemonte (2013).

Abd AL-Fattah et al. (2017) introduced the inverted Kumaraswamy (IKum) distribution and studied some of its properties. The maximum likelihood (ML) and Bayes estimators, confidence intervals for the parameters, the reliability and the hazard rate functions of the IKum distribution based on Type II censored samples, are obtained.

Assuming $T$ is a random variable distributed as IKum distribution with shape parameters; $\alpha>0$ and $\beta>0$, denoted by $T \sim \operatorname{IKum}(\alpha, \beta)$. Then the pdf, cdf, reliability function $(r f)$ and hazard rate function (hrf) are given, respectively, by

$$
\begin{aligned}
& f(t ; \alpha, \beta)=\alpha \beta(1+t)^{-(\alpha+1)}\left(1-(1+t)^{-\alpha}\right)^{\beta-1}, \quad t>0 ; \alpha, \beta>0, \\
& F(t ; \alpha, \beta)=\left(1-(1+t)^{-\alpha}\right)^{\beta}, \quad t>0 ; \alpha, \beta>0, \\
& R(t)=P(T>t)=1-F(t)=1-\left(1-(1+t)^{-\alpha}\right)^{\beta}, \quad t>0, \alpha, \beta>0,
\end{aligned}
$$

and

$h(t)=\frac{f(t)}{R(t)}=\frac{\alpha \beta(1+t)^{-(\alpha+1)}\left(1-(1+t)^{-\alpha}\right)^{\beta-1}}{1-\left(1-(1+t)^{-\alpha}\right)^{\beta}}, \quad t>0 ; \quad \alpha, \beta>0$.

Fatima et al. (2018) proposed the exponentiated IKum distribution; they derived some statistical properties of this distribution and used the ML method to estimate the parameters. Mohie El-Din and Abu-Moussa (2018) estimated the unknown parameters of the IKum distribution based on general progressive Type II censored data using ML and Bayesian methods. Also, ZeinEldin et al. (2019) introduced the Type I half-logistic IKum distribution, some statistical properties of this distribution are derived. The method of ML estimation, methods of least squares and weighted least squares estimation and method of Cramer-von Mises minimum distance estimation are used to estimate the parameters of this distribution. Usman and ul Haq (2020) introduced the Marshall-Olkin extended IKum distribution, sub models were showed of this generalization. They derived explicit expressions for major mathematical properties of this distribution and they estimated the parameters using the ML method.

This paper is organized as follows: In Section 2, ML estimators of the parameters, rf and hrf based on dgos are obtained. Bayes estimators of the parameters, $r f$ and $h r f$ based on dgos under squared error (SE) and linear exponential (LINEX) loss functions are derived in Section 3. Also, credible intervals for the parameters, $r f$ and $h r f$ are obtained. A numerical study is presented in Section 4.

\section{Maximum Likelihood Estimation Based on Dual Generalized Order Statistics}

In this section, the ML method is used to estimate the parameters, $r f$ and $h r f$ of the IKum distribution based on dgos. The asymptotic variance-covariance matrix of the ML estimators for the parameters $\alpha$ and $\beta$ and the asymptotic $100(1-\omega) \%$ confidence intervals for $\alpha$ and $\beta$ are obtained.

\subsection{Maximum likelihood estimation for the parameters}

Suppose that $T(1, n, m, k), T(2, n, m, k), \ldots, T(n, n, m, k)$ be $n$ dgos from IKum distribution, the likelihood function can be derived by substituting (2) and (3) in (1) as follows: 


$$
\begin{gathered}
L(\alpha, \beta ; \underline{t}) \propto\left[\prod_{i=1}^{n-1} \alpha \beta\left(1+t_{(i)}\right)^{-(\alpha+1)}\left(u_{i}\right)^{\beta m+\beta-1}\right]\left[\alpha \beta\left(1+t_{(n)}\right)^{-(\alpha+1)}\left(u_{n}\right)^{\beta k-1}\right] \\
=\alpha^{n} \beta^{n} \prod_{i=1}^{n}\left(1+t_{(i)}\right)^{-(\alpha+1)} \prod_{i=1}^{n-1}\left(u_{i}\right)^{\beta(m+1)-1}\left(u_{n}\right)^{\beta k-1}
\end{gathered}
$$

where $u_{n}=\left(1-\left(1+t_{(n)}\right)^{-\alpha}\right)$ and $u_{i}=\left(1-\left(1+t_{(i)}\right)^{-\alpha}\right)$.

The natural logarithm of the likelihood function is given by

$$
\begin{aligned}
\ell=\ln L(\alpha, \beta ; \underline{t})= & n \ln \alpha+n \ln \beta-(\alpha+1) \sum_{i=1}^{n} \ln \left(1+t_{(i)}\right) \\
& +[\beta(m+1)-1] \sum_{i=1}^{n-1} \ln \left(u_{i}\right)+(\beta k-1) \ln \left(u_{n}\right) .
\end{aligned}
$$

Considering that the two parameters $\alpha$ and $\beta$ are unknown and differentiating the log likelihood function partially in (8) with respect to $\alpha$ and $\beta$, one obtains

$\frac{\partial \ell}{\partial \beta}=\frac{n}{\beta}+(m+1) \sum_{i=1}^{n-1} \ln \left(u_{i}\right)+k \ln \left(u_{n}\right)$,

and

$$
\begin{aligned}
\frac{\partial \ell}{\partial \alpha}= & \frac{n}{\alpha}-\sum_{i=1}^{n} \ln \left(1+t_{(i)}\right)+(\beta(m+1)-1) \sum_{i=1}^{n-1} \frac{\left(1+t_{(i)}\right)^{-\alpha} \ln \left(1+t_{(i)}\right)}{u_{i}} \\
& +\frac{(\beta k-1)\left(1+t_{(n)}\right)^{-\alpha} \ln \left(1+t_{(n)}\right)}{u_{n}} .
\end{aligned}
$$

Equating the derivatives (9) and (10) to zero, one can obtain the ML estimator of $\beta$

$$
\hat{\beta}=\frac{-n}{(m+1) \sum_{i=1}^{n-1} \ln \left(1-\left(1+t_{(i)}\right)^{-\widehat{\alpha}}\right)+k \ln \left(1-\left(1+t_{(n)}\right)^{-\widehat{\alpha}}\right)} .
$$

Then the ML estimator of the parameter $\alpha$ can be obtained numerically by substituting (11) in (10).

\subsection{Maximum likelihood estimation for the reliability and hazard rate functions}

The invariance property of the ML estimation can be used to obtain the ML estimators $\hat{R}_{M L}\left(t_{0}\right)$ and $\hat{h}_{M L}\left(t_{0}\right)$, for a given time $t_{0}$, just replacing the parameters $\alpha$ and $\beta$ by their corresponding ML estimators, as given below

$$
\hat{R}_{M L}\left(t_{0}\right)=1-\left(1-\left(1+t_{0}\right)^{-\widehat{\alpha}}\right)^{\widehat{\beta}}, \quad t_{0}>0,
$$

and

$\hat{h}_{M L}\left(t_{0}\right)=\frac{\widehat{\alpha} \widehat{\beta}\left(1+t_{0}\right)^{-(\widehat{\alpha}+1)}\left(1-\left(1+t_{0}\right)^{-\widehat{\alpha}}\right)^{\widehat{\beta}-1}}{1-\left(1-\left(1+t_{0}\right)^{-\widehat{\alpha}}\right)^{\widehat{\beta}}}, t_{0}>0$.

\subsection{Asymptotic variance -covariance matrix of the maximum likelihood estimators}

The asymptotic variance -covariance matrix, $\boldsymbol{V}$, of the ML estimators for $\alpha$ and $\beta$ is the inverse of the observed Fisher information matrix, $F$, using the second derivatives of the logarithm of the likelihood function as follows:

$F \approx-\left[\begin{array}{cc}\frac{\partial^{2} \ell}{\partial \alpha^{2}} & \frac{\partial^{2} \ell}{\partial \alpha \partial \beta} \\ \frac{\partial^{2} \ell}{\partial \beta \partial \alpha} & \frac{\partial^{2} \ell}{\partial \beta^{2}}\end{array}\right]$,

and

$\boldsymbol{V}=F^{-1}$,

where 
$\frac{\partial^{2} \ell}{\partial \beta^{2}}=\frac{-n}{\beta^{2}}$,

$$
\begin{aligned}
\frac{\partial^{2} \ell}{\partial \alpha^{2}}= & \frac{-n}{\alpha^{2}}-\frac{(\beta k-1)\left(1+t_{(n)}\right)^{-2 \alpha} \ln \left(1+t_{(n)}\right)^{2}}{u_{n}^{2}}-\frac{(\beta k-1)\left(1+t_{(n)}\right)^{-\alpha} \ln \left(1+t_{(n)}\right)^{2}}{u_{n}} \\
& -(\beta(m+1)-1) \sum_{i=1}^{n-1}\left[\frac{\left(1+t_{(i)}\right)^{-2 \alpha} \ln \left(1+t_{(i)}\right)^{2}}{u_{i}^{2}}+\frac{\left(1+t_{(i)}\right)^{-\alpha} \ln \left(1+t_{(i)}\right)^{2}}{u_{i}}\right],
\end{aligned}
$$

and $\frac{\partial^{2} \ell}{\partial \beta \partial \alpha}=(m+1) \sum_{i=1}^{n-1} \frac{\left(1+t_{(i)}\right)^{-\alpha} \ln \left(1+t_{(i)}\right)}{u_{i}}+\frac{\left(1+t_{(n)}\right)^{-\alpha} \ln \left(1+t_{(n)}\right)}{u_{n}}$

The asymptotic normality of the ML estimation can be used to compute the two sided approximate

$100(1-\omega) \%$ confidence intervals for $\alpha$ and $\beta$ as follows:

$\hat{\alpha} \pm Z_{\left(1-\frac{\omega}{2}\right)} \sqrt{\tilde{\operatorname{var}(\hat{\alpha})}}$ and $\hat{\beta} \pm Z_{\left(1-\frac{\omega}{2}\right)} \sqrt{\widetilde{\operatorname{var}(\hat{\beta})}}$

Also, the asymptotic $100(1-\omega) \%$ confidence intervals for $r f$ and $h r f$ are given by

$\hat{R}_{M L}\left(t_{0}\right) \pm Z_{\left(1-\frac{\omega}{2}\right)} \sqrt{\widetilde{\operatorname{var}}\left(\hat{R}\left(t_{0}\right)\right)}$ and $\hat{h}_{M L}\left(t_{0}\right) \pm Z_{\left(1-\frac{\omega}{2}\right)} \sqrt{\widetilde{\operatorname{var}}\left(\hat{h}\left(t_{0}\right)\right)}$

where $Z_{\left(1-\frac{\omega}{2}\right)}$ is standard normal percentile and $\left(1-\frac{\omega}{2}\right)$ is the confidence coefficient.

\section{Bayesian Estimation Based on Dual Generalized Order Statistics}

The Bayesian approach is considered to estimate the parameters, $r f$ and $h r f$ of the IKum distribution based on dgos. The Bayes estimators are obtained under the SE and LINEX loss functions to estimate (point and credible intervals) of the parameters, $r f$ and $h r f$ of the IKum distribution based on $\boldsymbol{d g o s}$.

\subsection{Bayesian estimation under squared error loss function}

In this subsection, the Bayes estimators of the shape parameters, $r f$ and the $h r f$ based on dgos are obtained under SE loss function.

Assuming that the parameters $\alpha$ and $\beta$ of the IKum distribution are random variables with a joint bivariate prior density function that was considered by AL-Hussaini and Jaheen (1992) as

$\pi(\alpha, \beta)=\pi_{1}(\beta \mid \alpha) \pi_{2}(\alpha)$,

where $\quad \pi_{1}(\beta \mid \alpha)=\frac{\alpha^{\theta+1}}{\Gamma(\theta+1) w^{\theta+1}} \beta^{\theta} e^{\frac{-\alpha \beta}{w}}, \quad \theta>-1, w, \alpha, \beta>0$,

and the prior of $\alpha$ is

$$
\pi_{2}(\alpha)=\frac{\alpha^{c-1}}{\Gamma(c) b^{c}} e^{-\frac{\alpha}{b}}, \quad \alpha, b, c>0
$$

The joint prior pdf of $\alpha$ and $\beta$; will be obtained by substituting (20) and (21) in (19) as given below

$$
\pi(\alpha, \beta) \propto \alpha^{c+\theta} \beta^{\theta} e^{-\alpha\left(\frac{1}{b}+\frac{\beta}{w}\right)}, \quad c, b, w>0 .
$$

The joint posterior of $\alpha$ and $\beta$ can be derived by using (6) and (22) as follows:

$$
\begin{aligned}
& \pi(\alpha, \beta \mid \underline{t}) \propto L(\alpha, \beta \mid \underline{t}) \pi(\alpha, \beta) . \\
& =\frac{\left.\alpha^{n+\theta+c} \beta^{n+\theta} e^{-\alpha\left[\frac{1}{b}+\sum_{i=1}^{n} \ln (1+t\right.}(i)\right] e^{-\beta\left[\frac{\alpha}{w}-(m+1) \sum_{i=1}^{n-1} \ln \left(u_{i}\right)-k \ln \left(u_{n}\right)\right]}}{\prod_{i=1}^{n}\left(u_{i}\right)},
\end{aligned}
$$

hence, the joint posterior distribution of $\alpha$ and $\beta$ is given by

$\pi(\alpha, \beta \mid \underline{t})=\frac{\left.\left.\alpha^{n+\theta+c} \beta^{n+\theta} e^{-\alpha\left[\frac{1}{b}+\sum_{i=1}^{n} \ln (1+t\right.}(i)\right)\right] e^{-\beta\left[\frac{\alpha}{w}-(m+1) \sum_{i=1}^{n-1} \ln u_{i}-k \ln u_{n}\right]}}{\prod_{i=1}^{n} u_{i} \Gamma(n+\theta+1) \psi(\underline{t})}$,

where $u_{n}$ and $u_{i}$ are given by (7), also 
$\psi(\underline{t})=\int_{0}^{\infty} \frac{\alpha^{n+\theta+c} e^{-\alpha\left[\frac{1}{b}+\sum_{i=1}^{n} \ln (1+t(i))\right]}}{\prod_{i=1}^{n} u_{i}\left[\frac{\alpha}{w}-(m+1) \sum_{i=1}^{n-1} \ln u_{i}-k \ln u_{n}\right]^{n+\theta+1}} d \alpha$.

Under SE loss function the Bayes estimators for the parameters $\alpha, \beta, r f$ and $h r f$ are given, respectively, by their marginal posterior expectations using (25) as shown below

$$
\begin{aligned}
& \alpha_{(S E)}^{*}=E(\alpha \mid \underline{t})=\int_{0}^{\infty} \frac{\alpha^{n+\theta+c+1} e^{-\alpha\left[\frac{1}{b}+\sum_{i=1}^{n} \ln (1+t(i))\right]}}{\prod_{i=1}^{n} u_{i}\left[\frac{\alpha}{w}-(m+1) \sum_{i=1}^{n-1} \ln u_{i}-k \ln u_{n}\right]^{n+\theta+1} \psi(\underline{t})} d \alpha, \\
& \beta_{(S E)}^{*}=E(\beta \mid \underline{t})=\int_{0}^{\infty} \frac{(n+\theta+1) \alpha^{n+\theta+c} e^{-\alpha\left[\frac{1}{b}+\sum_{i=1}^{n} \ln \left(1+t t_{(i)}\right)\right]}}{\prod_{i=1}^{n} u_{i}\left[\frac{\alpha}{w}-(m+1) \sum_{i=1}^{n-1} \ln u_{i}-k \ln u_{n}\right]^{n+\theta+2} \psi(\underline{t})} d \alpha, \\
& R_{(S E)}^{*}(t)=E(R(t) \mid \underline{t})=1-\int_{0}^{\infty} \frac{\alpha^{n+\theta+c} e^{-\alpha\left[_{b}^{1}+\sum_{i=1}^{n} \ln \left(1+t_{(i)}\right)\right]}}{\prod_{i=1}^{n} u_{i}\left[\frac{\alpha}{w}-(m+1) \sum_{i=1}^{n-1} \ln u_{i}-k \ln u_{n}-\ln \left(1-(1+t)^{-\alpha}\right)\right]^{n+\theta+1} \psi(\underline{t})} d \alpha,
\end{aligned}
$$

and

$$
\begin{aligned}
& h_{(S E)}^{*}((t)=E(h(t) \mid \underline{t}) \\
& \quad=\int_{0}^{\infty} \int_{0}^{\infty} \frac{(1+t)^{-(\alpha+1)}\left(1-(1+t)^{-\alpha}\right)^{\beta-1} \alpha^{n+\theta+c+1} \beta^{n+\theta+1} e^{-\alpha\left[\frac{1}{b}+\sum_{i=1}^{n} \ln \left(1+t\left(t_{i}\right)\right]\right.} e^{-\beta\left[\frac{\alpha}{w}-(m+1) \sum_{i=1}^{n-1} \ln u_{i}-k \ln u_{n}\right]}}{\left[1-\left(1-(1+t)^{-\alpha}\right)^{\beta}\right] \prod_{i=1}^{n} u_{i} \Gamma(n+\theta+1) \psi(\underline{t})} d \alpha d \beta,
\end{aligned}
$$

To obtain the Bayes estimates of the parameters, $r f$ and $h r f$, (27)-(30) should be solved numerically.

Since, the posterior distribution is given by (25), then a $100(1-\omega) \%$ credible intervals for $\alpha$ and $\beta$ is $(L(\underline{t}), U(\underline{t}))$, respectively, where

$$
\begin{aligned}
& P[\alpha>L(\underline{t}) \mid \underline{t}]=\int_{L(\underline{t})}^{\infty} \frac{\alpha^{n+\theta+c} e^{-\alpha\left[\frac{1}{b}+\sum_{i=1}^{n} \ln \left(1+t_{(i)}\right)\right]}}{\prod_{i=1}^{n} u_{i}\left[\frac{\alpha}{w}-(m+1) \sum_{i=1}^{n-1} \ln u_{i}-k \ln u_{n}\right]^{n+\theta+1} \psi(\underline{t})} d \alpha=1-\frac{\omega}{2}, \\
& P[\alpha>U(\underline{t}) \mid \underline{t}]=\int_{U(\underline{t})}^{\infty} \frac{\alpha^{n+\theta+c} e^{-\alpha\left[\frac{1}{b}+\sum_{i=1}^{n} \ln (1+t(i))\right]}}{\prod_{i=1}^{n} u_{i}\left[\frac{\alpha}{w}-(m+1) \sum_{i=1}^{n-1} \ln u_{i}-k \ln u_{n}\right]^{n+\theta+1} \psi(\underline{t})} d \alpha=\frac{\omega}{2}, \\
& P[\beta>L(\underline{t}) \mid \underline{t}]=\int_{L(\underline{t})}^{\infty} \int_{0}^{\infty} \frac{\alpha^{n+\theta+c} \beta^{n+\theta} e^{-\alpha\left[\frac{1}{b}+\sum_{i=1}^{n} \ln (1+t(i))\right]} e^{-\beta\left[\frac{\alpha}{w}-(m+1) \sum_{i=1}^{n-1} \ln u_{i}-k \ln u_{n}\right]}}{\prod_{i=1}^{n} u_{i} \Gamma(n+\theta+1) \psi(\underline{t})} d \alpha d \beta=1-\frac{\omega}{2},
\end{aligned}
$$

and

$$
P[\beta>U(\underline{t}) \mid \underline{t}]=\int_{U(\underline{t})}^{\infty} \int_{0}^{\infty} \frac{\alpha^{n+\theta+c} \beta^{n+\theta} e^{-\alpha\left[\frac{1}{b}+\sum_{i=1}^{n} \ln (1+t(i))\right]} e^{-\beta\left[\frac{\alpha}{w}-(m+1) \sum_{i=1}^{n-1} \ln u_{i}-k \ln u_{n}\right]}}{\prod_{i=1}^{n} u_{i} \Gamma(n+\theta+1) \psi(\underline{t})} d \alpha d \beta=\frac{\omega}{2} .
$$

\subsection{Bayesian estimation under linear exponential loss function}

In this subsection, the Bayes estimators of the shape parameters, $r f$ and $h r f$ based on dgos are obtained under LINEX loss function. Under the LINEX loss function, the Bayes estimators for the shape parameters $\alpha, \beta, r f$ and $h r f$ are given, respectively, by

$$
\begin{aligned}
& \alpha_{(L N X)}^{*}=\frac{-1}{v} \ln E\left(e^{-v \alpha} \mid \underline{t}\right)=\frac{-1}{v} \ln \left[\int_{0}^{\infty} \frac{\alpha^{n+\theta+c} e^{-\alpha\left[v+\frac{1}{b}+\sum_{i=1}^{n} \ln (1+t(i))\right]}}{\prod_{i=1}^{n} u_{i}\left[\frac{\alpha}{w}-(m+1) \sum_{i=1}^{n-1} \ln u_{i}-k \ln u_{n}\right]^{n+\theta+1} \psi(\underline{t})} d \alpha\right], \\
& \beta_{(L N X)}^{*}=\frac{-1}{v} \ln E\left(e^{-v \beta} \mid \underline{t}\right)=\frac{-1}{v} \ln \left[\int_{0}^{\infty} \frac{\alpha^{n+\theta+c} e^{-\alpha\left[\frac{1}{b}+\sum_{i=1}^{n} \ln \left(1+t_{(i)}\right)\right]}}{\prod_{i=1}^{n} u_{i}\left[v+\frac{\alpha}{w}-(m+1) \sum_{i=1}^{n-1} \ln u_{i}-k \ln u_{n}\right]^{n+\theta+1} \psi(\underline{\underline{t}})} d \alpha\right], \\
& R_{(L N X)}^{*}(t)=\frac{-1}{v} \ln E\left(e^{-v R(t)} \mid \underline{t}\right)
\end{aligned}
$$




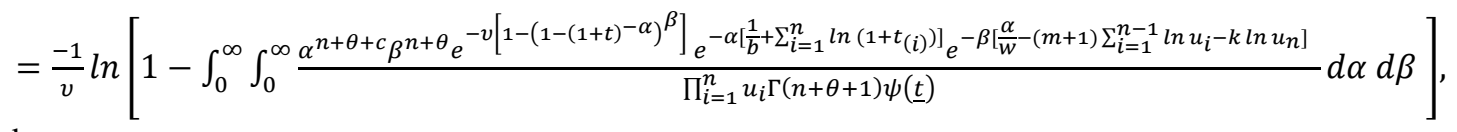

and

$h_{(L N X)}^{*}(t)=\frac{-1}{v} \ln E\left(e^{-v h(t)} \mid \underline{t}\right)$

$=\frac{-1}{v} \ln \left[\int_{0}^{\infty} \int_{0}^{\infty} \frac{\alpha^{n+\theta+c} \beta^{n+\theta} e^{-v \frac{\alpha \beta(1+t)^{-(\alpha+1)}(1-(1+t)-\alpha)^{\beta-1}}{1-\left(1-(1+t)^{-\alpha}\right) \beta}} e^{-\alpha\left[\frac{1}{b}+\sum_{i=1}^{n} \ln (1+t(i))\right]} e^{-\beta\left[\frac{\alpha}{w}-(m+1) \sum_{i=1}^{n-1} \ln u_{i}-k \ln u_{n}\right]}}{\prod_{i=1}^{n} u_{i} \Gamma(n+\theta+1) \psi(\underline{t})} d \alpha d \beta\right]$.

To obtain the Bayes estimates of the parameters, $r f$ and hrf, (35)-(38) should be solved numerically.

\section{Numerical Results}

This section aims to illustrate the theoretical results of the ML and Bayesian estimation under SE and LINEX loss functions. Numerical results are presented for the IKum distribution based on lower record values through a simulation study and some applications.

\subsection{Simulated example}

The lower record values can be obtained as a special case from dgos by setting $m=-1, k=1$; the estimation results obtained in Sections 2 and 3 can be specialized to lower records. The ML and Bayes estimates of $\alpha, \beta, r f$ and $h r f$ and their average estimates and Estimated Risks (ERs) are computed based on lower record values through Monte Carlo simulation study according to the following steps:

a. The population parameter values of $\alpha$ and $\beta$ are used to generate random samples of size $n$ from the IKum distribution observing that if $\mathrm{U}$ is uniform distribution $(0,1)$, then

$t_{i j}=\left[\left(1-u_{i j}^{\frac{1}{\beta}}\right)^{-\frac{1}{\alpha}}-1\right]$, is IKum $(\alpha, \beta)$ distribution.

b. For each sample size $\mathrm{n}$, consider the first observation is the first lower record value $t_{1}$ denoting it by $R_{1}$ and the second observation $t_{2}$ denoting it by $R_{2}$ which is smaller than the maximum record $\left(t_{1}>t_{2}\right)$ and if $t_{1} \leq$ $t_{2}$ ignore it and repeat until you get a sample of record values $(R v)$.

c. For the number of the surviving units $t$ and the population parameter values of the shape parameters, the ML estimates of the parameters $\alpha$ and $\beta$ are obtained, also the $r f$ and $h r f$ are calculated using the ML estimates of the parameters. The computations are performed using Mathematica 9.

d. The Bayes estimates of the parameters, $r f$ and $h r f$ under SE and LINEX loss functions are computed for the number of the surviving units $\mathrm{t}$ based on the population parameter values of the shape parameters $\alpha$ and $\beta$ and the hyper parameters of the prior distribution. The computations are performed using $\mathrm{R}$ programming language.

e. Tables 1 and 2 show the ERs of the estimates and 95\% confidence intervals of the shape parameters $\alpha$ and $\beta$ from the IKum distribution based on lower records where the population parameter values are $\alpha=1.1,1.2$, $\beta=1.5,2$ based on samples of $R v=3,5,7,9$ and replications $(N R)=2000$.

f. Table 3 displays the ML averages and 95\% confidence intervals of the $r f$ and $h r f$ at $t_{0}=0.5,1$, from the IKum distribution based on lower records for different samples of $R v$, and $N R=2000$.

g. Tables 4 and 5 present the Bayes estimates under informative prior of the parameters and their ERs, averages and credible intervals based on lower record values for different population parameter values for $\alpha=1.2,1.1$ and $\beta=0.8,0.4$, respectively, based on samples of $R v=5,7,9$ and $N R=10000$.

h. Table 6 displays the Bayes averages and 95\% confidence intervals of the $r f$ and $h r f$ at $t_{0}=0.5,1$, from the IKum distribution based on lower record values for different samples of $R v=5,9$, and $N R=10000$.

\subsection{Applications}

In this subsection, three applications to real data sets are provided to illustrate the importance of the IKum distribution based on lower records. Table 7 displays ML averages of the parameters, $r f, h r f$ and ERs from IKum distribution for the real data based on lower records. The averages of the Bayes estimates for the parameters, their ERs and the credible intervals based on informative prior are given in Table 8. To check the validity of the fitted model, 
Kolmogorov-Smirnov goodness of fit test is performed for each data set and the $\mathrm{p}$ values in each case indicates that the model fits the data very well.

I. The first application is a real data set obtained from Hinkley (1977). It consists of thirty successive values of March precipitation (in inches) in Minneapolis/St Paul. The data is 0.77, 1.74, 0.81, 1.20, 1.95, 1.20, 0.47, 1.43, $3.37,2.20,3.00,3.09,1.51,2.10,0.52,1.62,1.31,0.32,0.59,0.81,2.81,1.87,1.18,1.35,4.75,2.48,0.96,1.89$, $0.90,2.05$.

II. The second application is given by Murthy et al. (2004). The data refers to the time between failures for repairable items. The data is $1.43,0.11,0.71,0.77,2.63,1.49,3.46,2.46,0.59,0.74,1.23,0.94,4.36,0.40,1.74,4.73,2.23$, $0.45,0.70,1.06,1.46,0.30,1.82,2.37,0.63,1.23,1.24,1.97,1.86,1.17$.

III. The third application is the vinyl chloride data obtained from clean upgrading, monitoring wells in mg/L; this data set was used by Bhaumik et al. (2009). The data is: 5.1, 1.2, 1.3, 0.6, 0.5, 2.4, 0.5, 1.1, 8.0, 0.8, 0.4, 0.6, 0.9, $0.4,2.0,0.5,5.3,3.2,2.7,2.9,2.5,2.3,1.0,0.2,0.1,0.1,1.8,0.9,2.0,4.0,6.8,1.2,0.4,0.2$.

\subsection{Concluding remarks}

- From Tables 1 and 2 one can observe that the ERs of the ML averages for the shape parameters $\alpha$ and $\beta$ decreases when the sample size of lower records $R v$ increases. Also, the lengths of the confidence intervals become narrower as the sample of record size increases.

- It is clear from Tables 4 and 5 that the ERs of the Bayes averages for the parameters, $r f$ and $h r f$ perform better and the length of the credible intervals get shorter when the sample of $R v$ increases.

- One can notice that the ERs for the averages of the parameters, $r f$ and $h r f$ under LINEX loss function have the less values than the corresponding ERs of the averages under SE loss function.

Table 1: ERs of ML estimates and $95 \%$ confidence intervals of the shape parameters $\alpha$ and $\beta$ from IKum distribution based on lower records

$(\alpha=1.1, \beta=1.5, \mathrm{NR}=2000)$

\begin{tabular}{|c|c|c|c|c|c|}
\hline $\boldsymbol{R v}$ & Estimators & ER & LL & UL & Length \\
\hline & $\widehat{\boldsymbol{\alpha}}$ & 0.0019 & 0.7200 & 1.5457 & 0.8257 \\
3 & $\widehat{\boldsymbol{\beta}}$ & 0.0004 & 0.8860 & 2.3585 & 1.4725 \\
\hline & $\widehat{\boldsymbol{\alpha}}$ & 0.0010 & 0.7623 & 1.5013 & 0.7389 \\
5 & $\widehat{\boldsymbol{\beta}}$ & 0.0002 & 0.9326 & 2.1526 & 1.2200 \\
\hline & $\widehat{\boldsymbol{\alpha}}$ & 0.0009 & 0.7717 & 1.5017 & 0.7299 \\
7 & $\widehat{\boldsymbol{\beta}}$ & 0.0001 & 1.0639 & 1.9879 & 0.9240 \\
\hline & $\widehat{\boldsymbol{\alpha}}$ & 0.0008 & 0.7589 & 1.4678 & 0.7088 \\
9 & $\widehat{\boldsymbol{\beta}}$ & 0.0001 & 1.0519 & 1.8693 & 0.8173 \\
\hline
\end{tabular}


Table 2: ERs of ML estimates and 95\% confidence intervals of the shape parameters $\alpha$ and $\beta$ from IKum distribution based on lower records $(\alpha=1.2, \beta=2, \mathrm{NR}=2000)$

\begin{tabular}{|c|c|c|c|c|c|}
\hline $\boldsymbol{R v}$ & Estimators & ER & LL & UL & Length \\
\hline & $\widehat{\boldsymbol{\alpha}}$ & 0.0001 & 0.3488 & 1.5464 & 1.1977 \\
3 & $\widehat{\boldsymbol{\beta}}$ & 0.0003 & 1.2829 & 2.3881 & 1.1051 \\
\hline & $\widehat{\boldsymbol{\alpha}}$ & 0.0002 & 0.4058 & 1.5577 & 1.1519 \\
5 & $\widehat{\boldsymbol{\beta}}$ & 0.0001 & 1.2662 & 2.2125 & 0.9462 \\
\hline & $\widehat{\boldsymbol{\alpha}}$ & 0.0001 & 0.4053 & 1.5527 & 1.1473 \\
7 & $\widehat{\boldsymbol{\beta}}$ & 0.0001 & 1.3465 & 2.1038 & 0.7573 \\
\hline & $\widehat{\boldsymbol{\alpha}}$ & 0.0001 & 0.4369 & 1.5349 & 1.0979 \\
9 & $\widehat{\boldsymbol{\beta}}$ & 0.0001 & 1.3208 & 2.0244 & 0.7036 \\
\hline
\end{tabular}

Table 3: ML averages and 95\% confidence intervals of the $r f$ and $h r f$ at $t_{0}=0.5,1$, from IKum distribution based on lower records for different sample size of $R v$ and $N R=2000$

\begin{tabular}{|c|c|c|c|c|c|c|}
\hline $\boldsymbol{R v}$ & $\boldsymbol{t}_{\mathbf{0}}$ & Estimators & Average & LL & UL & Length \\
\hline \multirow{3}{*}{5} & \multirow{2}{*}{$\mathbf{0 . 5}$} & $\widehat{\boldsymbol{R}}\left(\boldsymbol{t}_{\mathbf{0}}\right)$ & 0.8832 & 0.7813 & 0.9853 & 0.2039 \\
\cline { 2 - 7 } & & $\widehat{\boldsymbol{h}}\left(\boldsymbol{t}_{\mathbf{0}}\right)$ & 0.3278 & 0.0332 & 0.6225 & 0.5893 \\
\hline \multirow{3}{*}{9} & $\widehat{\mathbf{R}}\left(\boldsymbol{t}_{\mathbf{0}}\right)$ & 0.7159 & 0.8144 & 0.9900 & 0.1755 \\
& \multirow{2}{*}{$\mathbf{0 . 5}$} & $\widehat{\boldsymbol{h}}\left(\boldsymbol{t}_{\mathbf{0}}\right)$ & 0.3545 & 0.0185 & 0.5451 & 0.5266 \\
\cline { 2 - 7 } & \multirow{2}{*}{$\mathbf{1}$} & $\widehat{\boldsymbol{h}}\left(\boldsymbol{t}_{\mathbf{0}}\right)$ & 0.9022 & 0.7791 & 0.9766 & 0.1976 \\
& $\mathbf{1}$ & $\widehat{\boldsymbol{R}}\left(\boldsymbol{t}_{\mathbf{0}}\right)$ & 0.2818 & 0.0534 & 0.6292 & 0.5757 \\
\hline
\end{tabular}

Table 4: Bayes averages of the parameters and their estimated risks and credible intervals based on lower records $(\alpha=1.1, \beta=0.9, N R=10000)$

\begin{tabular}{|c|c|c|c|c|c|c|c|}
\hline$R v$ & $\begin{array}{c}\text { Loss } \\
\text { functions }\end{array}$ & Estimators & Average & ER & $\mathbf{L L}$ & UL & Length \\
\hline \multirow{4}{*}{5} & & $\alpha^{*}$ & 1.0952 & $5.09 \mathrm{e}-05$ & 1.0838 & 1.1040 & 0.0201 \\
\hline & SE & $\boldsymbol{\beta}^{*}$ & 0.9230 & $2.73 e-01$ & 0.8982 & 0.9430 & 0.0447 \\
\hline & & $\alpha^{*}$ & 1.1094 & 0.0001 & 1.0992 & 1.1156 & 0.0164 \\
\hline & LINEX & $\boldsymbol{\beta}^{*}$ & 0.9047 & 0.2548 & 0.8939 & 0.9130 & 0.0190 \\
\hline \multirow{4}{*}{7} & & $\overline{\alpha^{*}}$ & 1.1004 & $8.61 \mathrm{e}-06$ & 1.0929 & 1.1060 & 0.0131 \\
\hline & SE & $\boldsymbol{\beta}^{*}$ & 0.9017 & $2.52 \mathrm{e}-01$ & 0.8934 & 0.9056 & 0.0122 \\
\hline & & $\overline{\alpha^{*}}$ & 1.1028 & $1.29 \mathrm{e}-05$ & 1.0985 & 1.1064 & 0.0079 \\
\hline & LINEX & $\boldsymbol{\beta}^{*}$ & 0.9069 & $2.56 \mathrm{e}-01$ & 0.8974 & 0.9107 & 0.0132 \\
\hline \multirow{4}{*}{9} & & $\boldsymbol{\alpha}^{*}$ & 1.0997 & $1.01 \mathrm{e}-07$ & 1.0995 & 1.0999 & 0.0005 \\
\hline & SE & $\boldsymbol{\beta}^{*}$ & 0.9000 & $2.50 \mathrm{e}-01$ & 0.8997 & 0.9001 & 0.0004 \\
\hline & & $\boldsymbol{\alpha}^{*}$ & 1.0999 & $2.63 \mathrm{e}-08$ & 1.0997 & 1.1002 & 0.0005 \\
\hline & LINEX & $\boldsymbol{\beta}^{*}$ & 0.8999 & $2.49 \mathrm{e}-01$ & 0.8996 & 0.9003 & 0.0006 \\
\hline
\end{tabular}


Table 5: Bayes averages of the parameters and their estimated risks and credible intervals based on lower records $(\alpha=1.2, \beta=0.8, N R=10000)$

\begin{tabular}{|c|c|c|c|c|c|c|c|}
\hline$R v$ & $\begin{array}{c}\text { Loss } \\
\text { functions }\end{array}$ & Estimators & Average & ER & $\mathbf{L L}$ & $\mathbf{U L}$ & Length \\
\hline \multirow{4}{*}{5} & & $\alpha^{*}$ & 1.1035 & 0.0921 & 1.0957 & 1.1093 & 0.0135 \\
\hline & SE & $\boldsymbol{\beta}^{*}$ & 0.8998 & 0.0901 & 0.8962 & 0.9034 & 0.0071 \\
\hline & & $\alpha^{*}$ & 1.1058 & 0.0936 & 1.0994 & 1.1110 & 0.0115 \\
\hline & LINEX & $\boldsymbol{\beta}^{*}$ & 0.8972 & 0.0917 & 0.8888 & 0.9039 & 0.0151 \\
\hline \multirow{4}{*}{7} & & $\alpha^{*}$ & 1.1005 & 0.0904 & 1.0989 & 1.1018 & 0.0029 \\
\hline & SE & $\boldsymbol{\beta}^{*}$ & 0.9011 & 0.0893 & 0.8991 & 0.9028 & 0.0036 \\
\hline & & $\boldsymbol{\alpha}^{*}$ & 0.1014 & 0.0908 & 1.0997 & 1.1023 & 0.0026 \\
\hline & LINEX & $\boldsymbol{\beta}^{*}$ & 0.8983 & 0.0910 & 0.8971 & 0.9000 & 0.0029 \\
\hline \multirow{4}{*}{9} & & $\boldsymbol{\alpha}^{*}$ & 1.1004 & 0.0902 & 1.0993 & 1.1010 & 0.0017 \\
\hline & SE & $\boldsymbol{\beta}^{*}$ & 0.8982 & 0.0910 & 0.8970 & 0.8992 & 0.0022 \\
\hline & & $\alpha^{*}$ & 1.1008 & 0.0904 & 1.0997 & 1.1015 & 0.0017 \\
\hline & LINEX & $\boldsymbol{\beta}^{*}$ & 0.9007 & 0.0895 & 0.8990 & 0.9016 & 0.0026 \\
\hline
\end{tabular}

Table 6: Bayes averages and credible intervals of the $\mathrm{rf}$ and hrf at $t_{0}=0.5,1$, from IKum distribution based on lower records for different sample size of recods $R v$, and repetitions $N R=10000$

\begin{tabular}{|c|c|c|c|c|c|c|}
\hline $\mathbf{R v}$ & $t_{0}$ & Estimators & Average & LL & UL & Length \\
\hline \multirow{2}{*}{5} & 0.5 & $\begin{array}{l}\widehat{\boldsymbol{R}}\left(\boldsymbol{t}_{\mathbf{0}}\right) \\
\widehat{\boldsymbol{h}}\left(\boldsymbol{t}_{\mathbf{0}}\right)\end{array}$ & $\begin{array}{l}0.9078 \\
0.5986\end{array}$ & $\begin{array}{c}0.8904 \\
0.59758\end{array}$ & $\begin{array}{l}0.9203 \\
0.5995\end{array}$ & $\begin{array}{l}0.0299 \\
0.0019\end{array}$ \\
\hline & 1 & $\begin{array}{l}\widehat{R}\left(t_{0}\right) \\
\widehat{h}\left(t_{0}\right)\end{array}$ & $\begin{array}{l}0.8843 \\
0.6009\end{array}$ & $\begin{array}{l}0.8707 \\
0.5995\end{array}$ & $\begin{array}{l}0.9005 \\
0.6022\end{array}$ & $\begin{array}{l}0.0298 \\
0.0026\end{array}$ \\
\hline \multirow{2}{*}{9} & 0.5 & $\begin{array}{l}\widehat{\boldsymbol{R}}\left(\boldsymbol{t}_{0}\right) \\
\widehat{\boldsymbol{h}}\left(\boldsymbol{t}_{0}\right)\end{array}$ & $\begin{array}{l}0.9094 \\
0.5987\end{array}$ & $\begin{array}{c}0.8986 \\
0.59737\end{array}$ & $\begin{array}{l}0.9177 \\
0.5996\end{array}$ & $\begin{array}{l}0.0191 \\
0.0023\end{array}$ \\
\hline & 1 & $\begin{array}{l}\widehat{R}\left(t_{0}\right) \\
\widehat{h}\left(t_{0}\right)\end{array}$ & $\begin{array}{l}0.9029 \\
0.6002\end{array}$ & $\begin{array}{l}0.8880 \\
0.5986\end{array}$ & $\begin{array}{l}0.9105 \\
0.6013\end{array}$ & $\begin{array}{l}0.0225 \\
0.0026\end{array}$ \\
\hline
\end{tabular}


Table 7: ML averages of the parameters, rf, hrf and estimated risks from IKum distribution for the real data based on lower records

\begin{tabular}{|c|c|c|c|c|}
\hline Real data & Rv & Estimators & Average & ER \\
\hline I & 3 & $\begin{array}{c}\widehat{\boldsymbol{\alpha}} \\
\widehat{\boldsymbol{\beta}} \\
\widehat{\boldsymbol{R}}(\boldsymbol{t}) \\
\widehat{\boldsymbol{h}}(\boldsymbol{t})\end{array}$ & $\begin{array}{l}1.3242 \\
2.1875 \\
0.6721 \\
0.4698\end{array}$ & $\begin{array}{l}0.5245 \\
0.9753 \\
0.0028 \\
0.0424\end{array}$ \\
\hline II & 5 & $\begin{array}{c}\widehat{\alpha} \\
\widehat{\boldsymbol{\beta}} \\
\widehat{\boldsymbol{R}}(t) \\
\widehat{\boldsymbol{h}}(t)\end{array}$ & $\begin{array}{l}0.8318 \\
2.1696 \\
0.8330 \\
0.2318\end{array}$ & $\begin{array}{l}0.0537 \\
0.9402 \\
0.0115 \\
0.0011\end{array}$ \\
\hline III & 7 & $\begin{array}{c}\widehat{\alpha} \\
\widehat{\beta} \\
\widehat{\boldsymbol{R}}(\boldsymbol{t}) \\
\widehat{\boldsymbol{h}}(\boldsymbol{t})\end{array}$ & $\begin{array}{l}0.5282 \\
1.2893 \\
0.7822 \\
0.2144\end{array}$ & $\begin{array}{l}0.0052 \\
0.0079 \\
0.0031 \\
0.0024\end{array}$ \\
\hline
\end{tabular}

Table 8: Bayes averages of the parameters, estimated risks and credible intervals for the real data based on lower records

\begin{tabular}{|c|c|c|c|c|c|c|c|c|}
\hline \multirow{2}{*}{$\begin{array}{l}\text { Real } \\
\text { data }\end{array}$} & \multirow{2}{*}{$\mathbf{R v}$} & \multirow{2}{*}{$\begin{array}{c}\text { Loss } \\
\text { functions }\end{array}$} & \multirow{2}{*}{ Estimators } & \multirow{2}{*}{ Average } & \multirow{2}{*}{ ER } & \multicolumn{3}{|c|}{ Credible interval } \\
\hline & & & & & & $\mathbf{L L}$ & UL & Length \\
\hline \multirow{4}{*}{ I } & \multirow{4}{*}{3} & & $\alpha^{*}$ & 1.1005 & 0.0406 & 1.0999 & 1.1024 & 0.0024 \\
\hline & & SE & $\boldsymbol{\beta}^{*}$ & 0.9003 & 0.0910 & 0.8967 & 0.8998 & 0.0031 \\
\hline & & & $\alpha^{*}$ & 1.1025 & 0.0399 & 1.0982 & 1.1010 & 0.0027 \\
\hline & & LINEX & $\boldsymbol{\beta}^{*}$ & 0.8993 & 0.0889 & 0.8997 & 0.9038 & 0.0041 \\
\hline \multirow{4}{*}{ II } & \multirow{4}{*}{2} & & $\boldsymbol{\alpha}^{*}$ & 1.0997 & $4.90 \mathrm{e}-01$ & 1.0996 & 1.1009 & 0.0013 \\
\hline & & SE & $\boldsymbol{\beta}^{*}$ & 0.8987 & $5.01 \mathrm{e}-07$ & 0.8985 & 0.9004 & 0.0018 \\
\hline & & & $\boldsymbol{\alpha}^{*}$ & 1.0998 & $4.90 \mathrm{e}-01$ & 1.0997 & 1.1013 & 0.0016 \\
\hline & & LINEX & $\boldsymbol{\beta}^{*}$ & 0.9003 & $1.63 \mathrm{e}-06$ & 0.8999 & 0.9022 & 0.0023 \\
\hline \multirow{4}{*}{ III } & \multirow{4}{*}{7} & & $\alpha^{*}$ & 1.1002 & $4.90 \mathrm{e}-01$ & 1.0993 & 1.1009 & 0.0016 \\
\hline & & SE & $\boldsymbol{\beta}^{*}$ & 0.8991 & $8.24 \mathrm{e}-07$ & 0.8983 & 0.8998 & 0.0014 \\
\hline & & & $\boldsymbol{\alpha}^{*}$ & 1.1002 & $4.90 \mathrm{e}-01$ & 1.0991 & 1.1009 & 0.0017 \\
\hline & & LINEX & $\boldsymbol{\beta}^{*}$ & 0.9000 & $5.30 \mathrm{e}-07$ & 0.8988 & 0.9016 & 0.0027 \\
\hline
\end{tabular}




\section{Acknowledgements}

The authors would like to thank Referees and the Editor for their constructive comments and corrections which led to improvements of an earlier version of this article.

\section{References}

1. Abd AL-Fattah, A. M., EL-Helbawy A. A. and AL-Dayian, G. R. (2017). Inverted Kumaraswamy distribution: properties and estimation. Pakistan Journal of Statistics, 33(1), 37-61.

2. Ahsanullah, M. (2004). A characterization of the uniform distribution by dual generalized order statistics, Communications in Statistics-Theory and Methods, 33(12), 2921-2928.

3. AL-Hussaini, E. K. and Jaheen, Z. F. (1992). Bayesian estimation of the parameters, reliability and failure rate functions of the Burr type XII failure model. Journal of Statistical Computation and Simulation, 41, 3140

4. Athar, H. and Faizan, M. (2011). Moments of lower generalized order statistics from power function distribution and its characterization. International Journal of Statistical Sciences, 11, 125-134.

5. Barreto-Souza, W. and Lemonte, A. J. (2013). Bivariate Kumaraswamy distribution: properties and a new method to generate bivariate classes. Statistics 47 (6), 1321-1342.

6. Bhaumik, D. K., Kapur, K. and Gibbons, R. D. (2009). Testing parameters of a gamma distribution for small samples. Technometrics, 51, 326-334.

7. Burkschat, M., Cramer, E. and Kamps, U. (2003). Dual generalized order statistics. International Journal of Statistics, LX1 (1), 13-26.

8. Cordeiro, G. M, Ortega, E. M. M. and Nadarajah, S. (2010). The Kumaraswamy Weibull distribution with application to failure data. Journal of the Franklin Institute, 347 (8), 1399-1429.

9. Fatima. K., Jan. U and Ahmad. S. P. (2018). Statistical properties and applications of the exponentiated inverted Kumaraswamy distribution. Journal of Reliability and Statistical Studies, 11(1), 93-102.

10. Hinkley, D. (1977). On quick choice of power transformations, Journal of the Royal Statistical Society Series C (Applied Statistics), 26 (1), 67-69.

11. Jaheen, Z. F. and Al Harbi, M. M. (2011). Bayesian estimation based on dual generalized order statistics from the exponentiated Weibull model. Journal of Statistical Theory and Applications, 10 (4), 591-602.

12. Jamal, F., Nasir, M. A., Ozel. G. and Elgarhy, M. (2019). Generalized inverted Kumaraswamy generated family of distributions: theory and applications. Journal of Applied Statistics, 46(16):2927-2944

13. Kamps, U. (1995). A concept of generalized order statistics. Journal of Statistical Planning and Inference, 48, 1-23.

14. Khaledi, B. E. and Kochar, S. (2005). Dependence orderings for generalized order statistics. Statistics and Probability Letters, 73(4), 357-367.

15. Khan, M. J. S. and Khan, M. L. (2012). Conditional expectation of function of dual generalized order statistics an alternative approach. ProbStat Forum, 5, 80-85.

16. Khan, R. U. and Kumar, D. (2010). On moments of lower generalized order statistics from exponentiated Pareto distribution and its characterization. Applied Mathematical Sciences, 4(55), 2711-2722.

17. Kim, C., Song, S. and W. Kim (2016). Statistical inference for Burr Type III distribution on dual generalized order statistics and real data analysis. Applied Mathematical Sciences, 10 (14), 683-695.

18. Kumaraswamy, P. (1980). A generalized probability density function for double bounded random processes. Journal of Hydrology, 46, 79-88.

19. Kumari, T. and Pathak, A. (2014). Conditional expectation of certain distributions of dual generalized order statistics. International Journal of Mathematical Analysis, 8 (3), 141-148.

20. Mahdizadeh, M. (2016). Reconstruction of the missing order statistics in the Topp-Leone distribution. International Journal of Statistics \& Economics 17 (3), 12-19.

21. Mahmoud, M. A. W., Abdel-Aty, A., Mohamed, N. M. and Hamedani, G. G. (2014). Recurrence relations for moments of dual generalized order statistics from Weibull Gamma distribution and its characterizations. Journal of Statistics Applications and Probability, 3 (2), 189-199.

22. MirMostafaee, S. M. T. K, Mahdizadeh, M. and Nadarajah, S. (2015). The beta Lindley distribution. Journal of Data Science 13 (3), 603-626.

23. MirMostafaee, S. M. T. K, Mahdizadeh, M. and Aminzadeh, M. (2016). Bayesian inference for the ToppLeone distribution based on lower k-record values. Japan Journal of Industrial and Applied Mathematics 33 (3), 637-669. 
24. MirMostafaee, S. M. T. K, Mahdizadeh, M. and Lemonte, A. J. (2017). The Marshall-Olkin extended generalized Rayleigh distribution: Properties and applications. Communications in Statistics: Theory and Methods 46 (2), 653-671.

25. Mohie El-Din, M and Abu-Moussa, M. (2018). On Estimation and prediction for the inverted Kumaraswamy distribution based on general progressive censored samples. Pakistan Journal of Statistics and Operation Research, XIV (3), 717-736.

26. Muhammed, H. Z. (2020). On a bivariate generalized inverted Kumaraswamy distribution. Physica A: Statistical Mechanics and its Applications, Elsevier, 553(C).

27. Murthy, D. N. P., Xie, M. and Jiang, R. (2004). Weibull Models. Wiley series in probability and statistics, John Wiley and Sons, New York.

28. Tavangar, M. (2011). Power function distribution characterized by dual generalized order statistics, Journal of Iranian Statistical Scoiety, 10(1), 13-27.

29. Usman, R. M. and ul Haq, M. A. (2020). The Marshall-Olkin extended inverted Kumaraswamy distribution: Theory and applications. Journal of King Saud University - Science, 32, 356-365.

30. ZeinEldin, R. A., Chesneau, C., Jamal, F. and Elgarhy, M. (2019). Statistical Properties and Different Methods of Estimation for Type I Half Logistic Inverted Kumaraswamy Distribution. Mathematics, 7(10), 1002. 